\title{
Optimal Design of PID Controller for Deep Space Antenna Positioning Using Weighted Cultural Artificial Fish Swarm Algorithm
}

Ahmed Tijani Salawudeen ${ }^{1}$, Bashir Muhammed Mu'azu', Yusuf Abubakar Sha'aban ${ }^{2}$ and Chung Jin Chan²*

${ }^{1}$ Department of Electrical and Computer Engineering, Ahmadu Bello University, Zaria, Nigeria

${ }^{2}$ Department of Math and Computer Science, Lawrence Technological University, Southfield Michigan, USA

\begin{abstract}
This paper proposed a modification of the Artificial Fish Swarm Algorithm (AFSA) using adaptive behaviour base combination of normative and situational knowledge inherent in cultural algorithm. Four variations (wCAFSA Ns, wCAFSA_Sd, wCAFSA_NsSd and wCAFSA_NsNd) of the AFSA called weighted Cultural Artificial Fish Swarm Algorithm (WCAFSA) were then proposed with the hope of reducing the chance of falling into local minima by the standard AFSA. The performances of these were first evaluated using a collection of seven optimizations benchmark functions. Thereafter, all the variants were used to design an optimized PID controller for the dc motor of deep space antenna azimuth position control with the hope of minimizing the cost function and maximizing the steady state responses. Simulation results demonstrated the effectiveness of the algorithms in determining efficiently the optimized PID controller gains. Even though all the developed algorithms demonstrated their effectiveness, the wCAFSA_NsSd appears to be more efficient.
\end{abstract}

Keywords: Artificial fish swarm algorithm; Cultural algorithm; PID controller; Antenna azimuth; DC motor

\section{Introduction}

Currently, the attention of Artificial Intelligent (IA) algorithms paradigm has shifted towards biological systems. This is due to the appealing characteristics exhibited by biological systems such as: cooperative communication, adaptability, sophistication, robustness etc. [1]. These appealing characteristics of biological systems present an obvious motivation for several researchers to propose a mechanism of Natural Evolution (NE) in an attempt to model AI algorithms whose characteristics can be compared to the characteristics of biological systems. Some of these algorithms include: Particle Swarm Optimization (PSO) [2], Artificial Fish Swarm Algorithms (AFSA) [3], Bacterial Foraging Algorithm (BFA) [4], Artificial Bee Colony Algorithm (ABC) [5], Cultural Algorithm (CA) [6] etc. Researchers have explored the capabilities of these algorithms through various applications in engineering, science and social sciences. However, there is no known single AI algorithm capable of solving all optimization problems. This is because, in theory, every optimization problem can be approximately modelled into mimicking particle behaviour of biological system. Due to the diversification and intensification capabilities of AFSA, this research focus on developing some new variations of AFSA using CA knowledge, adaptive parameters and cell formation classification.

AFSA is an optimization algorithm which was developed using the intelligent behaviour of swarm of fish [7]. In water, fish can search for areas with more food either by its own searching ability or by intelligently following the searching ability of its companion fish. Perhaps, the areas with more fish are mainly the areas with the most nutrition [8]. AFSA has demonstrated some important characteristics such as high convergence rate, insensitivity to initial values, flexibility, ease of implementation, robustness and high fault tolerance [9-12]. The individual behaviour of the artificial fish is to search for local minima. This makes it difficult to move towards the global solution individually, especially when dealing with tough optimization problems in engineering and related discipline. This has led to several liabilities such as inability to search extensively at later stage, inability to maintain the balance of exploration and exploitation which has a significant effect on the searching ability of the algorithm [13,14]. Several researches have shown that, parameters like step size and visual distance have demonstrated profound influence on the performance of AFSA. For example, AFSA has strong global searching ability when the visual distance is large and strong local searching ability when the visual distance is low. Also, the bigger the step size, the faster the convergence speed and vice versa $[7,15]$. Selecting these parameters suitable for a particular optimization problem domain is therefore a challenge for researchers. It is interesting to note that several researchers have presented various modifications in other to combat some of these shortcomings [7,10,16-18]. However, these methods have only improved the performance but not completely address the highlighted problems. Thus, in this paper, an adaptive behaviour based inertial weight was used alongside cultural algorithm and cell formation classification to propose new variations of improved cultural artificial fish swarm algorithm (wCAFSA).

Cultural Algorithm is a computational model derived from the model of cultural evolution process [6]. CA provides an explicit mechanism for global knowledge and an effective structure within which to model self-adaptation in evolutionary computation (EC) systems [19]. Several researches have shown that, cultural based biology evolution algorithms performed much better when compared with the original biological evolution process [10,19-22]. Hence, this paper proposed new variations of improved AFSA using both Situational and Normative in CA. These new variations are called weighted Cultural Artificial Fish Swarm Algorithm (wCAFSA). The performance of wCAFSA was initially evaluated using a total of applied mathematical optimization test functions [23]. Thereafter, the wCAFSAs were used

*Corresponding author: Chung Jin Chan, Department of Math and Computer Science, Lawrence Technological University, Southfield Michigan, USA, Tel: +1 248-204-4000; E-mail: cchung@It.edu

Received October 11, 2017; Accepted December 05, 2017; Published December 12, 2017

Citation: Salawudeen AT, Mu'azu BM, Sha'aban YA, Chan CJ (2017) Optima Design of PID Controller for Deep Space Antenna Positioning Using Weighted Cultural Artificial Fish Swarm Algorithm. J Electr Electron Syst 6: 243. doi: 10.4172/2332-0796.1000243

Copyright: (c) 2017 Salawudeen AT, et al. This is an open-access article distributed under the terms of the Creative Commons Attribution License, which permits unrestricted use, distribution, and reproduction in any medium, provided the original author and source are credited. 
Citation: Salawudeen AT, Mu'azu BM, Sha'aban YA, Chan CJ (2017) Optimal Design of PID Controller for Deep Space Antenna Positioning Using Weighted Cultural Artificial Fish Swarm Algorithm. J Electr Electron Syst 6: 243. doi: 10.4172/2332-0796.1000243

to design an optimized PID controller for deep space antenna azimuth position.

The remainder of this paper is structured as follows. The fundamentals of AFSA and CA necessary to understand their basic concept is presented in section two. The procedure and all the relevant concept use in developing the CAFSA is presented in section three. Section four discusses briefly the test function used to evaluate the performance of each of the algorithms. The PID controller objective function formulation and it implementation using the wCAFSAs is presented in section five. Simulation and discussion of results is presented in section six and section seven contains the conclusion.

\section{Artificial Fish Swarm and Cultural Algorithm}

\section{Artificial Fish Swarm Algorithm (AFSA)}

Assuming a minimization problem having D-dimensional search space, the state vector of the AFSA swarm is initialized with $\mathrm{N}$ population of artificial fish such that, the position of one artificial fish can be formulated as: $X_{i}=\left(x_{i l}, x_{i 2}, \ldots ., x_{i D}\right)$ for $. i=1,2 \ldots \ldots . N$ where $\mathrm{X}_{\mathrm{i}}$ is the status of the fish. This represents the direct variable for the objective function under consideration [14]. The presence of food concentration in the position of fish is expressed as a fitness function $y=f\left(x_{i}\right)$. The visual distance between the artificial fish is $d_{i, j}=\left\|X_{i}-X_{j}\right\|$, where $i$ and $j$ is a randomly generated fish. The step control parameter is the total number of the movement of artificial fish. The parameter $\delta$ is known as the degree of congestion (degree crowdedness).

Preying: Preying is the fundamental biological behaviour of fish in water. Generally, the fish intelligently sees the region with more food in water by vision or sense and moves quickly towards this region.

Suppose the initial position of AF is $X_{i}$, the AF chooses a state randomly within its visual such that [14]:

$$
X_{j}=X_{i}+\operatorname{rand}(0,1) \times \text { visual }
$$

Where $X_{j}$ is the new state and $X_{i}$ is the previous state

If $f\left(X_{j}\right)<f\left(X_{i}\right)$ in a minimization problem, it moves forward a step towards $X_{j}$ in the following direction $[23,24]$ :

$$
X_{i}^{(t+1)}=X_{i}^{(t)}+\operatorname{rand}(0,1) \times \operatorname{step} \times \frac{X_{j}^{(t)}-X_{i}^{(t)}}{\left\|X_{j}^{(t)}-X_{i}^{(t)}\right\|}
$$

Where $\left\|X_{j}^{(t)}-X_{i}^{(t)}\right\|=\left|\sqrt{\left(\left(X_{j}^{(t)}\right)^{2}-\left(X_{i}^{(t)}\right)^{2}\right)}\right|$ is the interval distance between artificial fish $j$ and artificial fish $i$.

If $f\left(X_{j}\right)>f\left(X_{i}\right)$, the artificial fish again selects another state randomly as its new position. If the $\mathrm{AF}$ cannot find a feasible solution within a given time, it moves one step randomly as shown in eqn. (3) $[7,11]$.

$$
X_{i}^{(t+1)}=X_{i}^{(t)}+\operatorname{rand}(0,1) \times \text { step }
$$

Swarming: In swarming, the fish will assemble and move in groups as a natural mechanism of guaranteeing their existence and avoiding danger. Suppose the present position of the artificial fish is $X_{i}$, and $n f$ are the number of fellow AFs within a particular visual, which is equal to the number of elements in a set of $B=\left\{X_{i} \mid d_{i j} \leq\right.$ visual $\}$. If $n f \neq 0$ the set $\mathrm{B}$ isnot an empty set. Let $X_{c}$ be the centre position and $Y_{c}$ the fitness of the centre position. Let $X_{c}=\sum_{j}^{\mathrm{nf}} \frac{X_{j}}{n f}$ and $Y_{c}=f\left(X_{c}\right)$. If $n f \times Y_{c}<\delta \times Y_{i}$, then this is not a crowded area. If $Y_{c}<Y_{i}$ the AF moves a step in the direction of the companions' centre position as follows [25].

$$
X_{i}^{(t+1)}=X_{i}^{(t)}+\operatorname{rand}(0,1) \times \operatorname{step} \times \frac{X_{c}^{(t)}-X_{i}^{(t)}}{\left\|X_{c}^{(t)}-X_{i}^{(t)}\right\|}
$$

If swarming is not advantageous, the AFSA executes the preying behaviour.

Chasing: When a fish finds food, neighbouring fish will trail and reach the food. Suppose the present position of the artificial fish is $X_{i}$, and $X_{m}$ denotes the best artificial fish within $X_{i}^{\prime}$ s visual distance. The parameter $n f$ is the number of $X_{m}$ 's within the visual distance. $n f \times Y_{m}<\delta \times Y_{i}$, if $Y_{m}<Y_{i}$ and $n f \times Y_{m}<\delta \times Y_{i}$, the artificial fish moves one step towards $X_{m}[7,11,25]$ :

$$
X_{i}^{(t+1)}=X_{i}^{(t)}+\operatorname{rand}(0,1) \times \operatorname{step} \times \frac{X_{m}^{(t)}-X_{i}^{(t)}}{\left\|X_{m}^{(t)}-X_{i}^{(t)}\right\|}
$$

If this is not advantageous, the AFSA also executes the behaviour of preying. Swarming influences few fish which confined in local extreme values to move in the direction of a few fishes moving towards the global extremum. Chasing accelerates AF movement towards a better state, and at the same time, speed up AF movement towards the best (global) extremum from the local extremum.

\section{Cultural Algorithm (CA)}

In CA, experience of individuals selected from the general population by the acceptance function is employed to generate problem solving knowledge that dwells in the belief space $[10,19]$. The general framework of CA is given in Figure 1.

From Figure 1, it can be observed that CA consists of dual inheritance structures which are the population space and the belief space. In this paper, the population space is made up of individual members of artificial fish. While the belief space is made of the best individuals selected from the population using the influence function.

Acceptance function: The acceptance function selects individual members and their performances that have the capability to influence the forming of the present belief space $[26,27]$. In this paper, the total

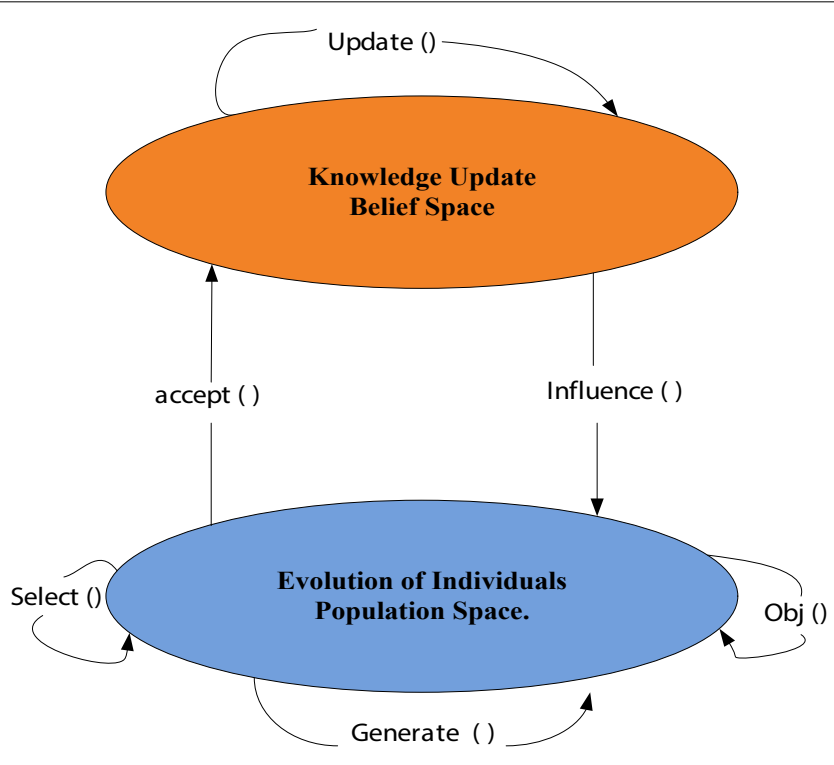

Figure 1: Framework of cultural algorithm. 
number of individuals which were accepted for updating the belief space is obtained using the following equation:

$$
f_{a}(N, t)=N . \beta+\lfloor N . \beta / t\rfloor
$$

Where $N$ is the population size, $t$ is the current number of generation and $\beta$ is chosen to be 0.2 (which means top $20 \%$ of the population) $[10,19,22]$. However, the entire population is selected at the beginning of the search processes and reduces to top $20 \%$ as the algorithm iterates.

Belief space structure: Five cultural knowledge have been identified in the belief space: Normative, Situational, Domain, History, and Topographical Knowledge [21]. In this paper, only normative and situational knowledge will be considered because they are deemed as fundamental for cultural evolution process.

Situational knowledge: Situational knowledge leads individuals to move towards direction of the best exemplars [19] and is the best individuals found so far in the solution space. In this paper, the situational knowledge is initialized using the best AFSA individuals as follows:

$$
S=\left\langle E^{t}\right\rangle=\left\langle S^{t} \mid S^{t}=\left\{s_{1}^{t}, s_{2}^{t}, \ldots, s_{n}^{t}\right\}\right\rangle,
$$

and is updated using:

$$
S_{j}^{t+1}=\left\{\begin{array}{ccc}
X_{\text {best }, j}^{t+1} & \text { if } & f\left(X_{\text {best }, j}^{t+1}\right)<f\left(s^{t}\right) \\
s_{j}^{t} & \text { otherwise }
\end{array},\right.
$$

Where $S$ store the exemplars and $E$ denote the exemplars themselves

$X_{\text {gbest }}^{t+1}$ represents the artificial fish with the best fitness in the swarm at generation $t+1$.

Normative knowledge: Normative knowledge is provide a set of likely variable limits that provide acceptable standards for individual traits and guidelines which provides the information for individual adjustments $[10,19,21,27]$. The normative knowledge in the belief space, $N$, is formally updated as follows:

$$
N=\langle I, U, L, D\rangle,
$$

where $U, L$ and $D$, are $\mathrm{n}$-dimensional vectors, and $I_{j}$ denotes the restricted interval for variable $j$, which is, a continuous set of real numbers $x$ delineated as follows:

$$
I_{j}=\left[l_{j}, u_{j}\right]=\left\{x \mid l_{j} \leq x \leq u_{j}\right\}
$$

$l_{j}$ and $u_{j}$ are the lower and upper bounds for the $j_{t h}$ variable, respectively, $L_{j}$ and $U_{j}$ (which are usually initialized with positive infinity) are the values of the objective function of the lower $\left(l_{j}\right)$ and upper $\left(u_{j}\right)$. The left boundary and its score for parameter $j$ is determined $[10,19,21,27]$, the interval update rule is formulated as:

$$
\begin{aligned}
& l_{j}^{t+1}=\left\{\begin{array}{cc}
x_{i, j}^{t} \quad \text { if } x_{i, j}^{t} \leq l_{j}^{t} \text { or } f\left(x_{i}^{t}\right)<L_{j}^{t} \\
l_{j}^{t} & \text { otherwise }
\end{array}\right. \\
& L_{j}^{t+1}=\left\{\begin{array}{cc}
f\left(X_{i}\right) & \text { if } x_{i, j}^{t} \leq l_{j}^{t} \text { or } f\left(x_{i}^{t}\right)<L_{j}^{t} \\
L_{j}^{t} & \text { otherwise }
\end{array}\right.
\end{aligned}
$$

Where $i^{\text {th }}$ individual affects the lower bound for parameter $j, l_{j}^{t}$ represents the lower limit of parameter $j$ at generation $t$ and $L_{j}^{t}$ denotes the fitness score of it. The right limits and the score for parameter $j$ also determine as:

$$
u_{j}^{t+1}=\left\{\begin{array}{cc}
x_{k, j} & \text { if } x_{k, j} \geq u_{j}^{t} \text { or } f\left(x_{k}\right)<U_{j}^{t} \\
u_{j}^{t} & \text { otherwise }
\end{array}\right.
$$

$$
U_{j}^{t+1}=\left\{\begin{array}{cc}
f\left(x_{k}\right) & \text { if } x_{k, j} \geq u_{j}^{t} \text { or } f\left(x_{k}\right)<U_{j}^{t} \\
U_{j}^{t} & \text { otherwise }
\end{array}\right.
$$

Where $\mathrm{k}^{\text {th }}$ artificial fish influence the upper bound for individual j. $u_{j}^{t}$ represents the upper limit for variable $j$ at generation $t$ and $U_{j}^{t}$ denotes the performance score for it. Every other information on CA can be found $[10,19,21,25,28]$.

\section{Weighted Cultural Artificial Fish Swarm Algorithm (wCAFSA)}

Since one of the major challenges of AFSA is the constant effects of its control parameters (visual, step and crowd). This paper introduced an iterative approach capable of appropriately selecting these parameters as follows

$$
\begin{aligned}
& \text { Visual }_{w}=\text { Visual }\left(\frac{w_{\max }-w_{\min }}{\text { iter }_{\max }}\right)^{n} \\
& \text { Visual }_{w}=\text { Visual } \times\left(\frac{w_{\max }-w_{\min }}{\text { iter }_{\max }}\right)^{n}
\end{aligned}
$$

Where

$w_{\max }$ and $w_{\text {win }}$ is the minimum and maximum values of two randomly generated numbers, $n$ is a factor of best and current individuals determined as follows:

$$
n=\frac{\text { gbest }- \text { current }}{\text { gbest }+ \text { current }}
$$

Eqn. (12) is used to determine the contribution factor of the previous best individual to the individual at the present time step.

\section{wCAFSA influence function}

This paper presents five variants of the proposed weighted cultural artificial fish swarm algorithm (wCAFSA_Ns, wCAFSA_Sd, wCAFSA_ NsSd, wCAFSA_NsNd and wCAFSA_SSd). The influence functions for these variants are discussed as follows.

wCAFSA_Ns: This variant employ only normative knowledge to influence the step movement of wCAFSA during evolution.

Preying_Ns: A selection operator was first introduced to initialize the wCAFSA_Ns. This operator is given as follows

$$
X_{i}^{t+1}=X_{i}^{t}+\operatorname{rand}(0,1) \times \operatorname{size}\left(I_{i}\right) \times \text { Visual }_{w}
$$

Where size $\left(I_{i}\right)=u_{i}-l_{i}$ is the size of the belief space interval of $\mathrm{i}^{\text {th }}$ parameter, which is determined by the normative knowledge for variable $i^{\text {th }}[10,19,21]$ and $r$ and $(0,1)$ is a random variable generated with a mean of zero and a deviation of 1 . The mathematical expression describing the preying behaviour of wCAFSA_Ns is given as follows:

$$
x_{i k}^{t+1}=x_{i k}^{t}+\frac{\operatorname{size}\left(I_{k}\right) \times \text { step }_{w} \times \operatorname{rand}(0,1) \times\left(x_{i k}^{t+1}-x_{i k}^{t}\right)}{\left\|X_{i}^{t+1}-X_{i}^{t}\right\|}
$$

Where $\operatorname{size}\left(I_{k}\right)=u_{k}-l_{k}$ the size of the belief space interval for the $k$ parameter and is also decided by normative knowledge for $k^{\text {th }}$ variable.

Swarming_Ns: The swarming behaviour of wCAFSA_Ns is described as follows.

$$
x_{i k}^{t+1}=x_{i k}^{t}+\frac{\operatorname{size}\left(I_{k}\right) \times \operatorname{step}_{w} \times \operatorname{rand}(0,1) \times\left(x_{c k}^{t}-x_{i k}^{t}\right)}{\left\|X_{c}^{t+1}-X_{i}^{t}\right\|}
$$

Where $X_{c}=\sum_{j=1}^{n f} X_{j} / n f$ is the centre position and $n f$ is the number of 
its fellow within the visual distance, $x_{c k}$ is the centre position associated with the upper bound for variable $j$.

Chasing_Ns: The chasing behaviour in this variant is described as follows:

$$
x_{i k}^{t+1}=x_{i k}^{t}+\frac{\operatorname{size}\left(I_{k}\right) \times \operatorname{step}_{w} \operatorname{rand}(0,1) \times\left(x_{m k}^{t}-x_{i k}^{t}\right)}{\left\|X_{m}^{t}-X_{i}^{t}\right\|}
$$

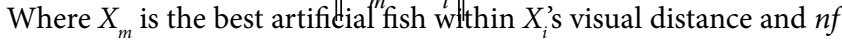
is the total number of $X_{i}$ 's fellows within the visual distance.

wCAFSA_Sd: In this variant, the situational knowledge is used to guide the direction of evolution of AFSA.

Preying_Sd: The preying behaviour of wCAFSA_Sd is described as follows:

$$
x_{i, k}^{t+1}=\left\{\begin{array}{l}
x_{i, k}^{t}+\left|\frac{\operatorname{Step}_{w} \times \operatorname{rand}(0,1) \times\left(x_{i, k}^{t+1}-x_{i, k}^{t}\right)}{\left\|X_{i}^{t+1}-X_{i}^{t}\right\|}\right| \\
x_{i, k}^{t}-\left|\frac{\operatorname{Step}_{w} \times \operatorname{rand}(0,1) \times\left(x_{i, k}^{t+1}-x_{i, k}^{t}\right) \mid}{\left\|X_{i}^{t+1}-X_{i}^{t}\right\|}\right| \\
\mid \text { if } x_{i, k}^{t}<E_{k}^{t}>E_{k}^{t} \\
x_{i, k}^{t}+\frac{\text { Step }_{w} \times \operatorname{rand}(0,1) \times\left(x_{i, k}^{t+1}-x_{i, k}^{t}\right)}{\left\|X_{i}^{t+1}-X_{i}^{t}\right\|} \text { otherwise }
\end{array}\right.
$$

Swarming_Sd: The swarming behaviour of the wCAFSA_Sd is as

follows

$$
x_{i, k}^{t+1}=\left\{\begin{array}{l}
x_{i, k}^{t}+\left|\frac{\operatorname{Step}_{w} \times \operatorname{rand}(0,1) \times\left(x_{c, k}^{t+1}-x_{i, k}^{t}\right)}{\left\|X_{c}^{t+1}-X_{i}^{t}\right\|}\right| \text { if } x_{i, k}^{t}<E_{k}^{t} \\
x_{i, k}^{t}-\left|\frac{\operatorname{Step}_{w} \times \operatorname{rand}(0,1) \times\left(x_{c, k}^{t+1}-x_{i, k}^{t}\right) \mid}{\left\|X_{c}^{t+1}-X_{i}^{t}\right\|}\right| \text { if } x_{i, k}^{t}>E_{k}^{t} \\
x_{i, k}^{t}+\frac{\text { Step }_{w} \times \operatorname{rand}(0,1) \times\left(x_{c, k}^{t+1}-x_{i, k}^{t}\right)}{\left\|X_{c}^{t+1}-X_{i}^{t}\right\|} \text { otherwise }
\end{array}\right.
$$

Chasing _Sd: The chasing behaviour of the wCAFSA_Sd is described as follows:

$$
x_{i, k}^{t+1}=\left\{\begin{array}{l}
x_{i, k}^{t}+\left|\frac{\operatorname{Step}_{w} \times \operatorname{rand}(0,1) \times\left(x_{m, k}^{t+1}-x_{i, k}^{t}\right)}{\left\|X_{m}^{t+1}-X_{i}^{t}\right\|}\right| \text { if } x_{i, k}^{t}<E_{k}^{t} \\
x_{i, k}^{t}-\left|\frac{\operatorname{Step}_{w} \times \operatorname{rand}(0,1) \times\left(x_{m, k}^{t+1}-x_{i, k}^{t}\right) \mid}{\left\|X_{m}^{t+1}-X_{i}^{t}\right\|}\right|
\end{array} \text { if } x_{i, k}^{t}>E_{k}^{t}\right.
$$

wCAFSA_NsSd: In this variant, both the normative and situational knowledge are integrated. The normative knowledge is used to guide the step movement while the situational knowledge is used to influence the direction of evolution.

Preying_NsSd: The preying behaviour of this variant is given as follows

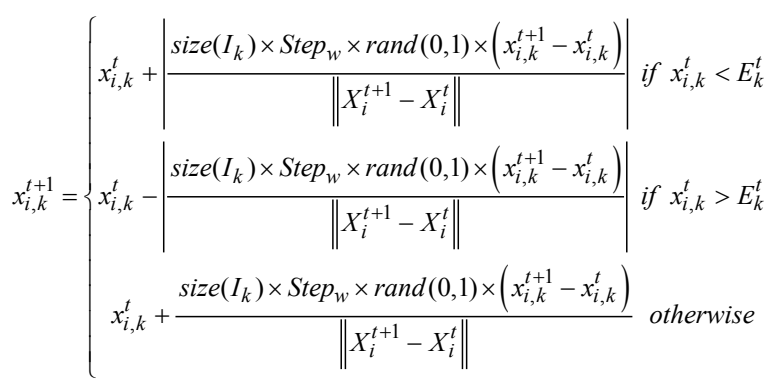

Swarming_NsSd: The swarming behaviour of this variant is presented as

$$
x_{i, k}^{t+1}=\left\{\begin{array}{l}
x_{i, k}^{t}+\left|\frac{\operatorname{size}\left(I_{k}\right) \times \operatorname{Step}_{w} \times \operatorname{rand}(0,1) \times\left(x_{c, k}^{t+1}-x_{i, k}^{t}\right)}{\left\|X_{c}^{t+1}-X_{i}^{t}\right\|}\right| \\
x_{i, k}^{t}-\left|\frac{\operatorname{size}^{t}\left(I_{k}\right) \times \operatorname{Step}_{w} \times \operatorname{rand}(0,1) \times\left(x_{c, k}^{t+1}-x_{i, k}^{t}\right)}{\left\|X_{c}^{t+1}-X_{i}^{t}\right\|}\right| \\
x_{i, k}^{t}+\frac{\operatorname{size}_{i, k}^{t}\left(I_{k}\right) \times \operatorname{Step}_{w} \times \operatorname{rand}(0,1) \times\left(x_{c, k}^{t+1}-x_{i, k}^{t}\right)}{\left\|X_{c}^{t+1}-X_{i}^{t}\right\|} \text { otherwise } x_{i, k}^{t}>E_{k}^{t}
\end{array}\right.
$$

Chasing_NsSd: The chasing behaviour of this variant is as follows

$$
x_{i, k}^{t+1}=\left\{\begin{array}{l}
x_{i, k}^{t}+\left|\frac{\operatorname{size}\left(I_{k}\right) \times \text { Step }_{w} \times \operatorname{rand}(0,1) \times\left(x_{m, k}^{t+1}-x_{i, k}^{t}\right)}{\left\|X_{c}^{t+1}-X_{i}^{t}\right\|}\right| \\
x_{i, k}^{t}-\left|\frac{\operatorname{size}^{t}\left(I_{k}\right) \times \operatorname{Step}_{w} \times \operatorname{rand}(0,1) \times\left(x_{m, k}^{t+1}-x_{i, k}^{t}\right) \mid}{\left\|X_{c}^{t+1}-X_{i}^{t}\right\|}\right|
\end{array} \text { if } x_{i, k}^{t}>E_{k}^{t}>E_{k}^{t}\right.
$$

wCAFSA_NsNd: The Swarming behaviour of AFSA is modified using the normative knowledge to direct the step size as well as direction of evolution as follows:

Preying_NsNd: The Preying behaviour of AFSA is modified using normative knowledge as follows:

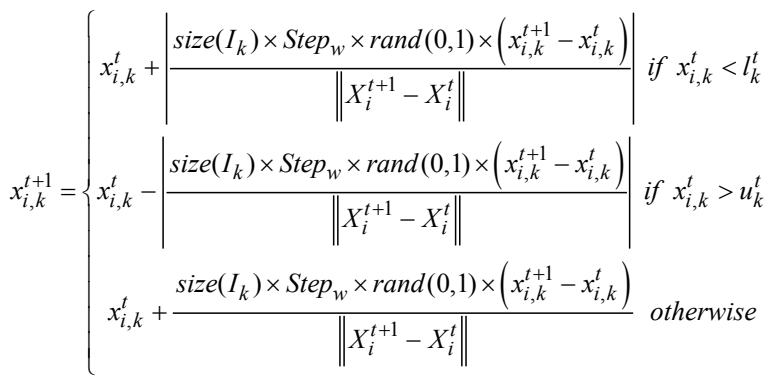

Swarming_NsNd: The Chasing behaviour of AFSA is modified using the normative knowledge as follows:

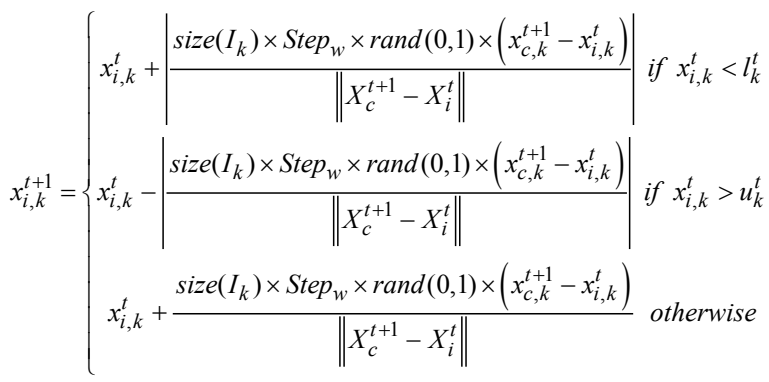


Chasing_NsNd: The Chasing behaviour of AFSA is modified using the normative knowledge as follows:

$$
x_{p+i, j}= \begin{cases}x_{i, k}+\left|\frac{\operatorname{Step}_{w} \times \operatorname{rand}(0,1) \times\left(x_{i, k}^{t+1}-E_{i, k}^{t}\right)}{\left\|X_{i, k}^{t+1}-E_{i, k}^{t}\right\|}\right| & \text { if } x_{i, k}<E_{k}^{t} \\ x_{i, k}-\left|\frac{\operatorname{Step}_{w} \times \operatorname{rand}(0,1) \times\left(x_{i, k}^{t+1}-E_{i, k}^{t}\right)}{\left\|X_{i, k}^{t+1}-E_{i, k}^{t}\right\|}\right| & \text { if } x_{i, k}>E_{k}^{t} \\ x_{i, k}+\frac{\operatorname{Step}_{w} \times \operatorname{rand}(0,1) \times\left(x_{i, k}^{t+1}-E_{i, k}^{t}\right)}{\left\|X_{i, k}^{t+1}-E_{i, k}^{t}\right\|} & \text { otherwise }\end{cases}
$$

wCAFSA_Ssd: This variant use situational knowledge to guide both the step size and direction of evolution if formulated as follows:

Preying_Ssd: The preying behaviour of this variant is given as

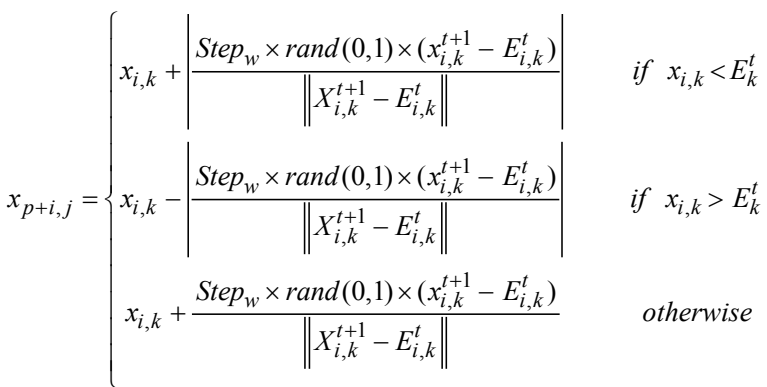

Swarming_Ssd

$$
x_{p+i, j}=\left\{\begin{array}{l}
x_{i, k}+\left|\frac{\operatorname{Step}_{w} \times \operatorname{rand}(0,1) \times\left(x_{c, k}^{t+1}-E_{i, k}^{t}\right)}{\left\|X_{c, k}^{t+1}-E_{i, k}^{t}\right\|}\right| \\
x_{i, k}-\left|\frac{\operatorname{Step}_{w} \times \operatorname{rand}(0,1) \times\left(x_{c, k}^{t+1}-E_{i, k}^{t}\right)}{\left\|X_{c, k}^{t+1}-E_{i, k}^{t}\right\|}\right| \\
x_{i, k}+\frac{\operatorname{Step}_{w} \times \operatorname{rand}(0,1) \times\left(x_{c, k}^{t+1}-E_{i, k}^{t}\right)}{\left\|X_{c, k}^{t+1}-E_{i, k}^{t}\right\|}
\end{array} \quad \text { if } x_{i, k}>E_{k}^{t}<E_{k}^{t}\right.
$$

Where $E_{k}^{t}$ is the best exemplar parameter value of the situational knowledge for variable $\mathrm{k}$ in the belief space. If an individual's parameter value is less than that of the current best, probably it would be better to go toward the current best. The present best individuals itself is mutated using a random direction, and is the otherwise case in all the equations.

\section{Test Function Evaluation}

Several test functions have been reported in the literature. However, there is no standard list or set of test functions one has to follow when choosing a subset for evaluating algorithm. Perhaps, any new or modified optimization algorithms should be tested using a collection of functions with diverse properties so as to verify the performance of the algorithm on different optimization problems efficiently [29]. In this paper, a total of seven mathematical nonlinear test functions are used to investigate the optimization capability of wCAFSA. These functions were selected due to their multimodality and its widespread usage. The functions adopted in this research include Ackley, Sphere, Levi and Montelvo, Rastrigin, Dejong Exponential and Schaffer [23,29,30].

\section{Optimized PID Controller Design}

In order to determine the efficiency of the wCAFSA, the algorithm was used to design an optimized PID controller for deep space antenna azimuth position control. The mathematical model and detail information of the armature DC servo motor controlling the antenna azimuth were adopted [31,32] follows:

$$
\frac{\theta_{o}(s)}{\theta_{i}(s)}=G(s)=\frac{6.63 K}{s^{3}+101.71 s^{2}+171 s+6.63 K}
$$

Where; $\mathrm{K}$ is the pre-amplifier gain.

The PID controller whose parameters are tuned by the wCAFSA was implemented using MATLAB R2015b simulation environment. The implementation procedure is discussed in the following section.

\section{Objective function formulation}

At the tuning stage, an objective function which is based on measured errors was formulated. Recall that the generalize equation of PID controller is [33-35],

$$
U(t)=K_{p} \times e(t)+\frac{1}{T_{i}} \int_{0}^{t} e(t) d t+T_{d} \frac{d e(t)}{d t}
$$

Where; $K_{p}$ is the proportional gain, $T_{i}$ is the integral time, $T_{d}$ is the derivative time.

The tracking error which is the difference between the desired output and the actual output is represented bye $(t)$. This error is sent to the PID controller and the controller compute its integral and derivative gains. The magnitude of the input signal from the controller is determined as follows $[33,36]$ :

$$
U(t)_{c}=K_{p} \times|e(t)|+K_{i} \times e(t)+K_{d} \times e(t)
$$

The objective function which is the PID performance criteria is formulated as a function of these errors (Integral Absolute Error-IAE, Integral of Time Square Error-ITSE and Integral of Square Error-ISE) as follow [36]:

$$
\begin{aligned}
I A E & =\int_{0}^{\infty}\left|r(t)-y(t) d t=\int_{0}^{\infty}\right| e(t)|d t| \\
I S E & =\int_{0}^{\infty} e^{2}(t) d t \\
I S E & =\int_{0}^{\infty} t \cdot e^{2}(t) d t
\end{aligned}
$$

Eqn. (35-37) show a time domain representation of the errors. An optimized value of the PID controller gains can obtain good system behaviour capable of minimizing the performance criteria in the time domain.

\section{wCAFSA implementation}

The developed wCAFSA variants (wCAFSA_Ns, wCAFSA_Sd, wCAFSA_NsSd and wCAFSA_NsNd) were applied to the objective function discussed in subsection 4.3 with the aim of minimizing the performance criterion. The performance criterion includes: Overshoot of the system (OS), Rise Time (RT), Settling Time (ST), Steady State Error (SSE) and Cost function. It has been stated that, for a good controller design, these performance criterions specifically the OS and ST should be less than $20 \%$ and 20 seconds, respectively $[33,37,38]$. The block diagram representation of the proposed PID tuning method is shown in Figure 2.

Unlike the conventional method of tuning PID controller, wCAFSA method (which in this case are the PID gains) having unfavourable costs are discarded and those with favourable cost are retained in the searching process. 
Citation: Salawudeen AT, Mu'azu BM, Sha'aban YA, Chan CJ (2017) Optimal Design of PID Controller for Deep Space Antenna Positioning Using Weighted Cultural Artificial Fish Swarm Algorithm. J Electr Electron Syst 6: 243. doi: 10.4172/2332-0796.1000243

Page 6 of 8

The wCAFSA block in Figure 2, contain the variant under evaluation, this could be any of the four (4) variants presented in this paper. Each wCAFSA simulation consists of three artificial fishes (which are the PID gains- Kp,Ki and $K d$ ). This means that, the search space consists of three dimensions and the artificial fishes must swarm in this three-dimensional space.

\section{Simulation and Results}

In order to demonstrate the effectiveness of the proposed tuning method, the responses of the system were analysed for two different preamplifier's gains, K. However, the choice of this gain should be carefully made and should depend on the output performance of the system response. Based on this assertion, the paper evaluates the performance of the optimized PID controller under a preamplifier gain of 50 and 100 respectively. This choice is pearly the authors and is carefully made after series of simulation.

\section{Simulation parameters}

There are no known standard formats for the selection of the control parameters of the artificial fish swarm algorithm $[7,14,18]$. However, for the purpose of this paper the parameters shown in Table 1 were employed.

Note that these parameters (except search dimension) can be varied under various simulations (PID gains) condition (Table 2). A search dimension of 40 was used when the performance of the developed wCAFSA algorithms were evaluated using the seven (7) benchmark functions. A search dimension of three (3), which corresponds to the PID gains, was used for the controller design $[3,7,11,14]$.

\section{Simulation results and discussion}

The optimized results obtained for each of the amplifier gains are discussed in this subsection. For an amplifier gain of 50, the responses of the system and cost function minimization under a unity feedback

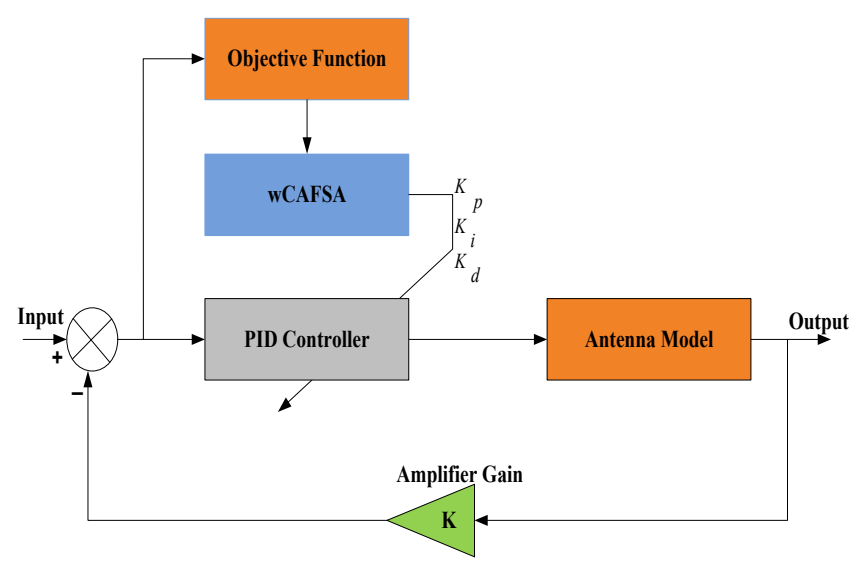

Figure 2: Implementation of system using PID Controller.

\begin{tabular}{|c|c|c|}
\hline SN & Parameters & Value \\
\hline $\mathbf{1}$ & Population of Fish & 80 \\
\hline $\mathbf{2}$ & Search Dimension & $40 ; 3$ \\
\hline $\mathbf{3}$ & Visual Distance & 5 \\
\hline $\mathbf{4}$ & Step Size & 0.75 \\
\hline $\mathbf{5}$ & Crowd Factor & 0.25 \\
\hline $\mathbf{6}$ & Number of Iteration & 100 \\
\hline $\mathbf{7}$ & Preamplifier Gain (K) & 50,100 \\
\hline
\end{tabular}

Table 1: Simulation parameters. are shown in Figures 3 and 4, respectively. The optimized PID controller gains and the steady state performance of the system are given in Table 3.

Figures 5 and 6 show the response of deep space antenna azimuth for an amplifier gain of 100 . The steady state performance is detailed in Table 2 .

From Table 3, it can be observed that, the performance of the system when the wCAFSA_NsSd based PID controller is used under an amplifier gain of 50 performed much better in terms of overshoot $(0.3788 \%)$ and settling time $(2.3876 \mathrm{sec})$ which are shown in bold italics. Similarly, for an amplifier gain of 100, the wCAFSA_NsSd also performed much better in terms of overshoot but wCAFSA_NsNd performed better in settling time $(2.1366 \mathrm{sec})$ in this case which is shown in bold. In general, the performance of the system under an amplifier gain of 50 is much better when compared to the performance of the system under an amplifier gain of 100. Even though the wCAFSA_NsSd based PID controller performed better than the other variants, the performance of all the other variants can also be considered efficient

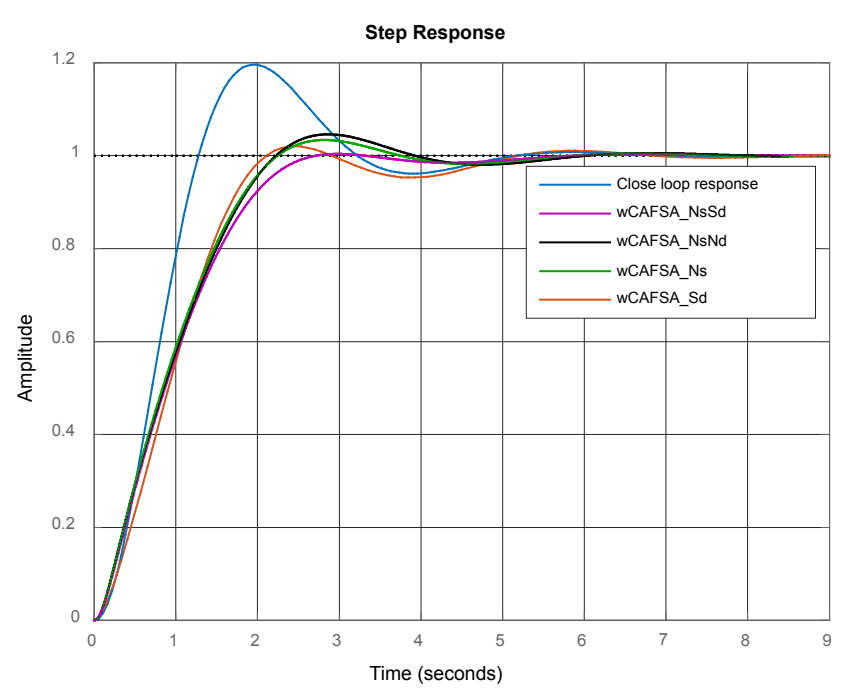

Figure 3: Step Response under an amplifier gain of 50

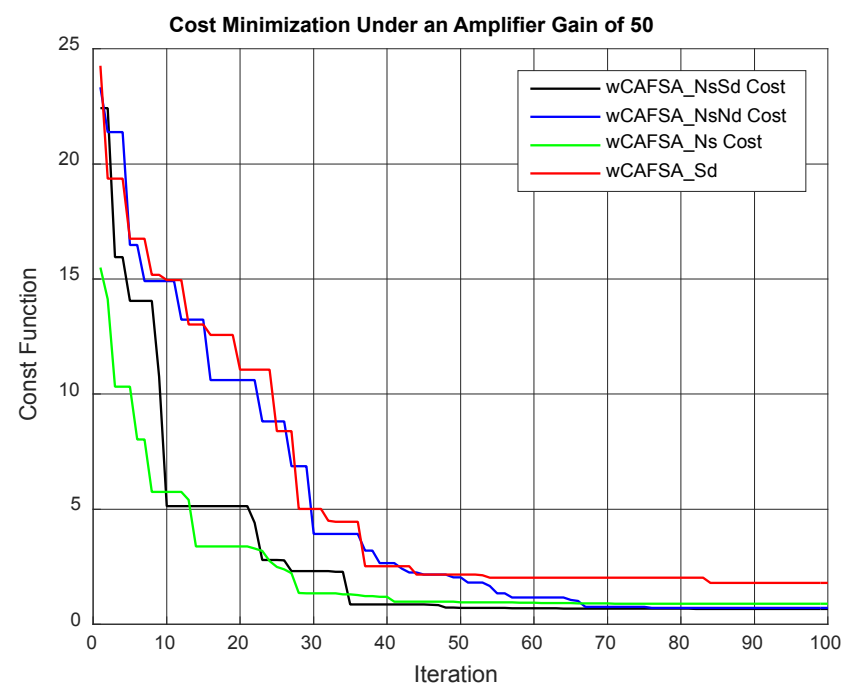

Figure 4: Cost minimization under an amplifier gain of 50 . 
Citation: Salawudeen AT, Mu'azu BM, Sha'aban YA, Chan CJ (2017) Optimal Design of PID Controller for Deep Space Antenna Positioning Using Weighted Cultural Artificial Fish Swarm Algorithm. J Electr Electron Syst 6: 243. doi: 10.4172/2332-0796.1000243

Page 7 of 8

\begin{tabular}{|c|c|c|c|c|c|c|c|}
\hline Function & AFSA & wCAFSA_Nd & wCAFSA_Sd & wCAFSA_NsSd & wCAFSA_NsNd & Best & Global \\
\hline Ackley & 0.6801 & $1.244 \mathrm{e}-3$ & 1.378 & $4.4409 \mathrm{e}-15$ & $8.3416 e-12$ & wCAFSA_NsSd & 0 \\
\hline Sphere & 1.0303 & 0.55255 & 0.83943 & $4.9407 e-324$ & $1.1124 \mathrm{e}-308$ & wCAFSA_NsSd & 0 \\
\hline LM1 & 0.7483 & 2.0457 & 0.64742 & $4.7116 \mathrm{e}-32$ & $7.7375 e-30$ & wCAFSA_NsSd & 0 \\
\hline Rastrigin & 231.330 & 135.736 & 37.9337 & 12.9345 & 38.1162 & wCAFSA_NsSd & 0 \\
\hline Dejong & $8.3052 e-8$ & $9.2032 \mathrm{e}-14$ & $3.4044 \mathrm{e}-12$ & $8.4254 e-63$ & $2.7874 \mathrm{e}-144$ & wCAFSA_NsNd & 0 \\
\hline Exp & 0 & 0 & 0 & 0 & 0 & Same & 0 \\
\hline Schaffer & 0.8771 & 5.8536 & 6.8536 & 0.57188 & 2.37 & wCASFA_NsSd & 0 \\
\hline
\end{tabular}

Table 2: wCAFSA performance evaluation on the benchmark functions.

\begin{tabular}{|c|c|c|c|c|c|c|c|c|c|}
\hline \multirow[b]{2}{*}{ SN } & \multirow[b]{2}{*}{ Parameters } & \multicolumn{2}{|c|}{ wCAFSA_NsSd } & \multicolumn{2}{|c|}{ wCAFSA_NsNd } & \multicolumn{2}{|c|}{ wCAFSA_Ns } & \multicolumn{2}{|c|}{ wCAFSA_Sd } \\
\hline & & $\mathrm{K}=50$ & $K=100$ & $\mathrm{~K}=50$ & $K=100$ & $\mathrm{~K}=50$ & $\mathrm{~K}=100$ & $\mathrm{~K}=50$ & $\mathrm{~K}=100$ \\
\hline 1 & $\mathrm{~K}_{\mathrm{p}}$ & 0.2862 & 0.0374 & 0.2382 & 0.0399 & 0.2674 & 0.0474 & 0.3035 & 0.0552 \\
\hline 2 & $\mathrm{~K}_{\mathrm{i}}$ & 1.01000 & 1.000 & 1.1090 & 1.1400 & 1.1050 & 1.1399 & 1.0050 & 1.0087 \\
\hline 3 & $K_{d}$ & 0.2063 & 0.2073 & 0.235 & 0.18073 & 0.2273 & 0.3021 & 0.1273 & 0.4311 \\
\hline 4 & RT (sec) & 1.6574 & 1.9385 & 1.5692 & 1.6009 & 1.5454 & 1.9367 & 1.3998 & 2.3567 \\
\hline 5 & ST (sec) & 2.3876 & 2.5100 & 3.5510 & 2.1366 & 3.3165 & 3.8556 & 4.7240 & 4.8721 \\
\hline 6 & OS (\%) & 0.3788 & 1.3455 & 4.5901 & 1.8514 & 3.3821 & 4.5269 & 2.0302 & 4.6325 \\
\hline 7 & Peak Time (sec) & 2.9763 & 3.0914 & 2.8631 & 2.6812 & 2.8180 & 3.1163 & 2.4524 & 3.8062 \\
\hline 8 & Peak & 1.0038 & 1.0135 & 1.0459 & 1.0185 & 1.0338 & 1.0453 & 1.0203 & 1.0363 \\
\hline
\end{tabular}

Table 3: Controller gain and steady state performance.

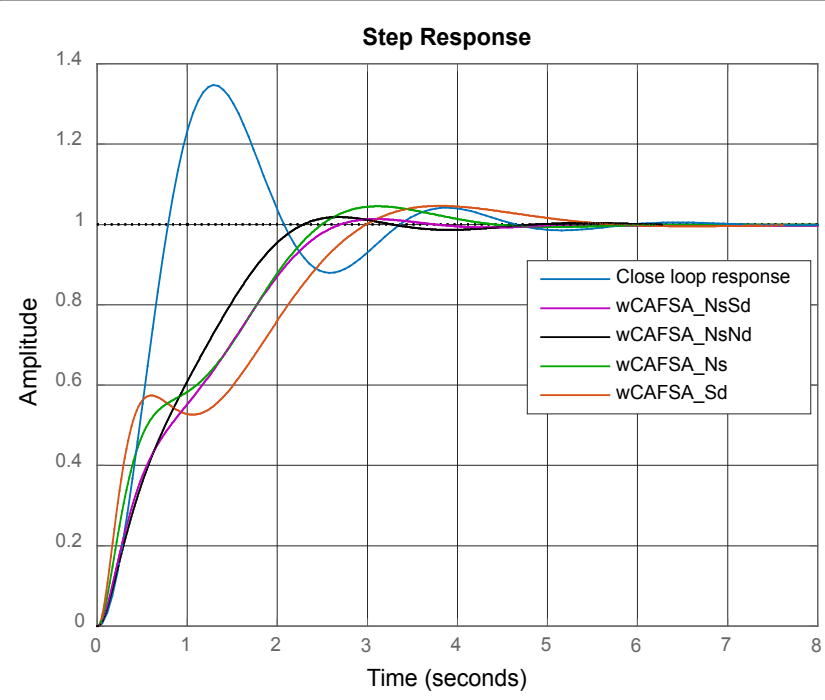

Figure 5: Step response under an amplifier gain of 100 .

and acceptable having settling time of less than 5 second and overshoot of less than 5\%. The details of the results can be found in Table 3 .

\section{Conclusion and Future Work}

This paper has presented a modified AFSA using the situational and normative knowledge inherent in cultural evolution process. Four variations using different configurations of the two knowledge were developed. The performance of the developed algorithm variants was evaluated using a total of seven (7) mathematical optimization test functions. The developed variants were used to design an optimized PID controller for deep space antenna azimuth positioning control. Simulation results demonstrate the efficiency of the proposed algorithms in determining the PID controller gains. The performance of the developed algorithms when applied to more complex models and the development of variations of the algorithm (using other forms of cultural knowledge) are the subjects of on-going research.

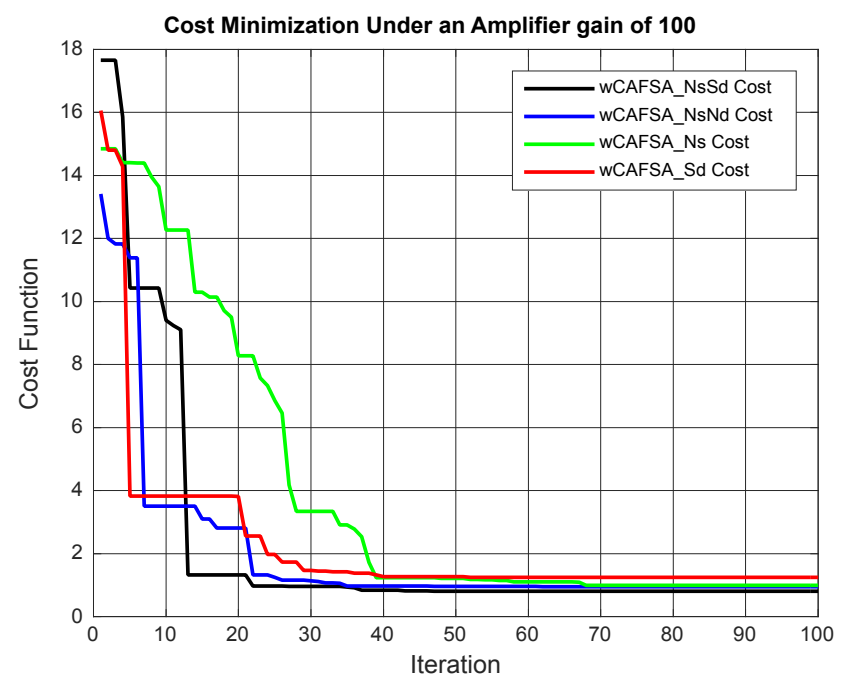

Figure 6: Cost minimization under an amplifier gain of 100 .

\section{References}

1. Xing B, Gao WJ (2014) Innovative Computational Intelligence: A Rough Guide to 134 Clever Algorithms. Computational Intelligence and Complexity.

2. Eberhart R, Kennedy J (1995) A New optimizer using particle swarm theory Proceedings of the Sixth International Symposium on Micro Machine and Human Science.

3. Li X, Shao Z, Qian J (2002) An Optimizing Method based on Autonomous Animate: Fish Swarm Algorithm. System Engineering Theory and Practice 22 32-38.

4. Passino KM (2002) Biomimicry of bacterial foraging for distributed optimization and control. IEEE Control Systems 22: 52-67.

5. Karaboga D (2005) An idea based on honey bee swarm for numerical optimization. Erciyes University, engineering faculty, computer engineering department.

6. Reynolds RG (1994) An introduction to cultural algorithms. Proceedings of the third annual conference on evolutionary programming, pp: 131-139.

7. Mu'azu MB, Salawudeen AT, Sikiru TH, Muhammad A, Abdu Al (2015) 
Citation: Salawudeen AT, Mu'azu BM, Sha'aban YA, Chan CJ (2017) Optimal Design of PID Controller for Deep Space Antenna Positioning Using Weighted Cultural Artificial Fish Swarm Algorithm. J Electr Electron Syst 6: 243. doi: 10.4172/2332-0796.1000243

Weighted Artificial Fish Swarm Algorithm with Adaptive Behaviour Based Linear Controller Design for Nonlinear Inverted Pendulum. Jounal of Engineering Research JER 20: 1-12.

8. Neshat M, Sepidnam G, Sargolzaei M, Toosi AN (2012) Artificial fish swarm algorithm: a survey of the state-of-the-art, hybridization, combinatorial and indicative applications. Artificial Intelligence Review 42: 965-997.

9. Azad MAK, Rocha AMA, Fernandes EM (2014) Improved binary artificial fish swarm algorithm for the 0-1 multidimensional knapsack problems. Swarm and Evolutionary Computation 14: 66-75.

10. Gao XZ, Wu Y, Wang X, Zenger K, Huang X (2013) A cultural Artificial Fishswarm Algorithm for optimization. ICIC Express Letters, Part B: Applications 4: 231-236.

11. Salawudeen AT, Mu'azu MB (2015) Stabilization of Inverted Pendulum System using Intelligent Linear Quadratic Regulator Controller. 7th International Joint Conference on Computational Intelligence, Lisbon Portugal.

12. Yang WH (2014) An improved artificial fish swarm algorithm and its application in multiple sequence alignment. Journal of Computational and Theoretical Nanoscience 11: 888-892.

13. Peng $Y$ (2011) An improved artificial fish swarm algorithm for optimal operation of cascade reservoirs. Journal of computers 6: 740-746.

14. Wu Y, Gao XZ, Zenger K (2011) Knowledge-based Artificial Fish-Swarm algorithm. IFAC World Congress, Milano.

15. Rani MGA, Marimuthu A, Kavitha (2014) Artificial fish swarm load balancing and job migration task with overloading detection in cloud computing environments. International Review on Computers and Software 9: 727-734.

16. Awad El-bayoumy M, Rashad M, Elsoud M, El-dosuky M (2013) FAFSA: Fast Artificial Fish Swarm Algorithm. International Journal of Information Science and Intelligent System 2: 60-70.

17. Wang Y, Li L (2015) An Improved Intelligent Algorithm Based On The Group Search Algorithm And The Artificial Fish Swarm Algorithm. Int J Optim Civil Eng 5: 37-52.

18. Yazdani D, Saman B, Sepas-Moghaddam A, Mohammad-Kazemi F, Reza Meybodi M (2013) A new algorithm based on improved artificial fish swarm algorithm for data clustering. International Journal of Artificial Intelligence 11 193-221.

19. Chung CJ (1997) Knowledge-based approaches to self-adaptation in cultural algorithms. Wayne State University.

20. Coelho LS, Mariani VC (2006) An efficient particle swarm optimization approach based on cultural algorithm applied to mechanical design. IEEE Congress on Evolutionary Computation, pp: 1099-1104.

21. Jin X, Reynolds RG (1999) Using Knowledge-Based Evolutionary Computation to Solve Nonlinear Constraint optimization problems: a cultural algorithm approach. Proceedings of the 1999 Congress on Evolutionary Computation.

22. Yin Z, Ye C, Yin W, Wen M (2011) A Culture Evolution Based on IWO Approach for DNA. Journal of Computational Information Systems 7: 5715-5722.
23. Li X, Tang K, Omidvar MN, Yang Z, Qin K, China H (2013) Benchmark functions for the CEC 2013 special session and competition on large-scale global optimization. Gene 7: 1-23.

24. Wu Y, Huang XL, Gao XZ, Zenger K (2012) Cultural artificial fish-swarm optimization algorithm and application in the parameters identification of roto system. Dianji yu Kongzhi Xuebao/Electric Machines and Control 16: 102-108.

25. Gao XZ, Wu Y, Zenger K, Huang X (2010) A knowledge-based Artificial Fishswarm Algorithm. 13th IEEE International Conference on Computational Science and Engineering, CSE, Hong Kong.

26. Reynolds RG, Chung CJ (1997) Fuzzy Approaches to Acquiring Experimental Knowledge. Ninth IEEE International Conference on Tools with Artificial Intelligence 97: 260-267.

27. Reynolds RG, Peng B (2004) Cultural Algorithms Modeling of How Culture Learn to Solve Problems. 16th IEEE International Conference on Tools with Artificial Intelligence.

28. Salawudeen AT (2015a) Development of an Improved Cultural Artificial Fish Swarm Algorithm with Crossover. Ahmadu Bello University Zaria, Nigeria.

29. Tang K, Yáo X, Suganthan PN, MacNish C, Chen YP, et al. (2007) Benchmark functions for the CEC'2008 special session and competition on large scale global optimization. Nature Inspired Computation and Applications Laboratory, USTC, China.

30. Zuo Y, Chen Y (2013) A novel particle swarm optimal algorithm hybridized with random disturbance term. Advanced Materials Research 765: 473-476.

31. Alwal LA, Kihato PK, Kamau SI (2016) DC Servomotor-based Antenna Positioning Control System Design using PID and LQR Controller. European International Journal of Science and Technology 5: 17-31.

32. Nise NS (2007) Control Systems Engineering. (With Cd): John Wiley \& Sons.

33. Allaoua B, Gasbaoui B, Mebarki B (2009) Setting up PID DC motor speed control alteration parameters using particle swarm optimization strategy. Leonardo Electronic Journal of Practices and Technologies 14: 19-32.

34. Allaoua B, Mebarki B (2012) Intelligent PID DC Motor Speed Control Alteration Parameters Using Particle Swarm Optimization. Artificial Intelligence Resources in Control and Automation Engineering 12: 3-14.

35. Ang KH, Chong G, Li Y (2005) PID control system analysis, design, and technology. IEEE Transactions on Control Systems Technology 13: 559-576.

36. Popov A, Farag A, Werner H (2005) Tuning of a PID controller using a multiobjective optimization technique applied to a neutralization plant. 44th IEEE Conference on Decision and Control.

37. Paulsen LM, Buckley MJ, West JB, Wolf JD (2016) Electronically steerable SATCOM antenna. US Patent 9337536 B1.

38. Pillai MRP, Jadhav SP, Patil DMD (2013) Tuning of PID Controllers using Advanced Genetic Algorithm. International Journal of Advanced Compute Science and Applications. 